\title{
Fluoxetine Affects Differentiation of Midbrain Dopaminergic Neurons In Vitro $\mathbf{s}$
}

\author{
Diana Lupu, ${ }^{1}$ Mukesh K. Varshney, ${ }^{1}$ Daniel Mucs, José Inzunza, Ulf Norinder, \\ Felicia Loghin, Ivan Nalvarte, ${ }^{2}$ and Joëlle Rüegg ${ }^{2}$
}

Department of Toxicology, Iuliu Haţieganu University of Medicine and Pharmacy, Cluj-Napoca, Romania (D.L., F.L.); Department of Biosciences and Nutrition, Karolinska Institutet, Huddinge, Sweden (M.K.V., J.I., I.N.); Unit of Work Environment Toxicology, Institute of Environmental Medicine (D.M.) and Department of Clinical Neuroscience (J.R.), Karolinska Institutet, Stockholm, Sweden; Department Computer and Systems Sciences, Stockholm University, Kista, Sweden (U.N.); and Swetox, Unit of Toxicology Sciences, Karolinska Institutet, Södertälje, Sweden (D.L., D.M., U.N., J.R.)

Received February 28, 2018; accepted July 11, 2018

\begin{abstract}
Recent meta-analyses found an association between prenatal exposure to the antidepressant fluoxetine (FLX) and an increased risk of autism in children. This developmental disorder has been related to dysfunctions in the brains' rewards circuitry, which, in turn, has been linked to dysfunctions in dopaminergic (DA) signaling. The present study investigated if FLX affects processes involved in dopaminergic neuronal differentiation. Mouse neuronal precursors were differentiated into midbrain dopaminergic precursor cells (mDPCs) and concomitantly exposed to clinically relevant doses of FLX. Subsequently, dopaminergic precursors were evaluated for expression of differentiation and stemness markers using quantitative polymerase chain reaction. FLX treatment led to increases in early regional specification markers orthodenticle homeobox 2 (Otx2) and homeobox engrailed-1 and -2 (En1 and En2). On the other hand, two
\end{abstract}

transcription factors essential for midbrain dopaminergic $(\mathrm{mDA})$ neurogenesis, LIM homeobox transcription factor $1 \alpha(L m \times 1 a)$ and paired-like homeodomain transcription factor 3 (Pitx3) were downregulated by FLX treatment. The stemness marker nestin (Nes) was increased, whereas the neuronal differentiation marker $\beta 3$-tubulin (Tubb3) decreased. Additionally, we observed that FLX modulates the expression of several genes associated with autism spectrum disorder and downregulates the estrogen receptors (ERs) $\alpha$ and $\beta$. Further investigations using ER $\beta$ knockout (BERKO) mDPCs showed that FLX had no or even opposite effects on several of the genes analyzed. These findings suggest that FLX affects differentiation of the dopaminergic system by increasing production of dopaminergic precursors, yet decreasing their maturation, partly via interference with the estrogen system.

\section{Introduction}

Fluoxetine (FLX) is the most commonly prescribed selective serotonin reuptake inhibitor (SSRI) and has been in clinical use since the late 1980s (Perez-Caballero et al., 2014). This highly lipophilic drug crosses the blood-brain barrier and selectively blocks the serotonin transporter in the brain, thus inhibiting serotonin reuptake from the synaptic cleft (PerezCaballero et al., 2014). This leads to increased serotonergic neurotransmission resulting in antidepressant effects (PerezCaballero et al., 2014). FLX is also widely prescribed to treat

This work was supported by the Swedish Research Council Formas [Grants 210-2012-1502, 216-2013-1966] and by Emil and Wera Cornell's research foundation.

${ }^{1}$ D.L. and M.K.V. contributed equally to this work.

${ }^{2}$ I.N. and J.R. contributed equally to this work.

https://doi.org/10.1124/mol.118.112342.

S This article has supplemental material available at molpharm.aspetjournals. depression during pregnancy, despite a lack of knowledge regarding both treatment efficacy and risks for the fetus and child (Pei et al., 2016). Although FLX passes through the placental barrier, little is known about how it affects (neuro)developmental outcomes in children exposed in utero (Rampono et al., 2009; Pei et al., 2016).

Several studies have found an association between antenatal SSRI exposure and increased risks for autism spectrum disorders (ASD) in children. Three recent systematic reviews and meta-analyses of large case-control and cohort studies found associations between in utero SSRI exposure and an increased risk (1.5- to 2-fold) for ASD (Man et al., 2015; Andalib et al., 2017; Kaplan et al., 2017) and two others found associations between maternal use of antidepressants (including SSRIs) during pregnancy and increased risk for ASD in children (Rais and Rais, 2014; Mezzacappa et al., 2017). A number of rodent studies have shown reduced play behavior

ABBREVIATIONS: AMPA, $\alpha$-amino-3-hydroxy-5-methyl-4-isoxazolepropionic acid; ANOVA, analysis of variance; ASD, autism spectrum disorder; BERKO, ER $\beta$ knockout; DA, dopaminergic; En1, homeobox engrailed-1; En2, homeobox engrailed-2; ER, estrogen receptor; FLX, fluoxetine; 5-HT, 5-hydroxytryptamine; Lmx1a, LIM homeobox transcription factor $1 \alpha$; mDA, midbrain dopaminergic; mDPC, midbrain dopaminergic precursor cell; mESC, mouse embryonic stem cell; Nes, nestin; NPC, neural precursor cell; Nkx2.2, NK2 homeobox 2; Otx2, orthodenticle homeobox 2; Pax3, paired box 3; Pitx3, paired-like homeodomain transcription factor 3; Reln, reelin; Rora, retinoic acid receptor-related orphan receptor- $\alpha$; SSRI, selective serotonin reuptake inhibitor; Th, tyrosine hydroxylase; Tubb3, $\beta 3$-tubulin; WT, wild-type. 
TABLE 1

Primers used for mouse cDNA

\begin{tabular}{|c|c|c|}
\hline & Forward & Reverse \\
\hline $36 B 4$ & GACACCCTCCAGGAAGCGA & GTGTTCGACAATGGCAGCAT \\
\hline$E R \alpha$ & CTGTCCAGCAGTAACGAGAAAG & CACAGTAGCGAGTCTCCTTGG \\
\hline$E R \beta$ & TGCCTGTAAACAGAGAGACCC & AGTCATTATGTCCTTGAATGCTTCT \\
\hline Tubb3 & TGTCATCCACCTTCATTGGC & AACATGGCTGTGAACTGCTC \\
\hline Nes & AGGGCAAATCTGGGAACTTG & AGGGTTTCCATCTGCAAAGG \\
\hline En1 & ACAGAGAAAGCGAGCAAGAG & TTTTTCCCCCATAGCACCTG \\
\hline En2 & AGGTTCCAAGACGCTATCAC & TTTGAGCACTCCATCCAAGG \\
\hline Otx2 & GTCTTATCTAAAGCAACCGCCT & AGTAAATGTCGTCCTCTCCCT \\
\hline Nkx2.2 & GTGATCGTTGCCAAATGACG & TGCTCAGGAGACGAAAAAGG \\
\hline Lmx1a & AGCGAGCCAAGATGAAGAAG & TTGTCTGAGCAGAAGTCAGC \\
\hline Pitx3 & ACGGCTCTCTGAAGAAGAAGC & CATGTCAGGGTAGCGATTCCTC \\
\hline$T h$ & ATTGGAGGCTGTGGTATTCG & ACTTTCAAAGCCCGAGACAG \\
\hline Slc6a3 & ATGTCTTCACACTGCTGGA & ATTGCTGGACACCGTAGAAC \\
\hline Tph2 & GTGACCCTGAATCCGCCTG & GGTGCCGTACATGAGGACT \\
\hline Slc6a4 & GTTGATGCTGCGGCTCAGATCT & GAAGCTCGTCATGCAGTTCACC \\
\hline Foxp2 & AAGGAGCAGTGTGGACTGTG & GCAGACTGGCATTTAGAGCTG \\
\hline Gria2 & GCCGAGGCGAAACGAATGA & CACTCTCGATGCCATATACGTTG \\
\hline Pax3 & CCGGGGCAGAATTACCCAC & GCCGTTGATAAATACTCCTCCG \\
\hline Rora & GTGGAGACAAATCGTCAGGAAT & TGGTCCGATCAATCAAACAGTTC \\
\hline Reln & TTACTCGCACCTTGCTGAAAT & CAGTTGCTGGTAGGAGTCAAAG \\
\hline
\end{tabular}

Foxp2, Forkhead box protein P2; Gria2, glutamate ionotropic receptor AMPA type subunit 2.

and social motivation following perinatal administration of SSRIs (fluoxetine and citalopram), which is suggestive of neurodevelopmental disorders with impaired social behaviors, as in ASD (Olivier et al., 2011; Rodriguez-Porcel et al., 2011; Simpson et al., 2011; Khatri et al., 2014).

ASD is a neurodevelopmental disorder characterized by deficient social communication and interaction, as well as repetitive behavioral and activity patterns (American Psychiatric Association, 2013). The causes of ASD have not been fully elucidated, but the etiology of this complex disorder is known to involve a combination of genetic and environmental factors that underlie dysfunctions in the amygdala, prefrontal cortex, and nucleus accumbens (Chaste and Leboyer, 2012; Park et al., 2016). These brain areas are associated with social behavior and social

\section{A}

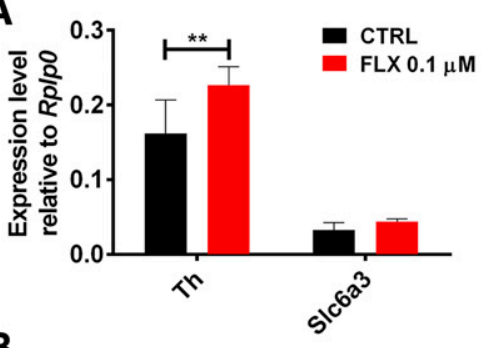

B
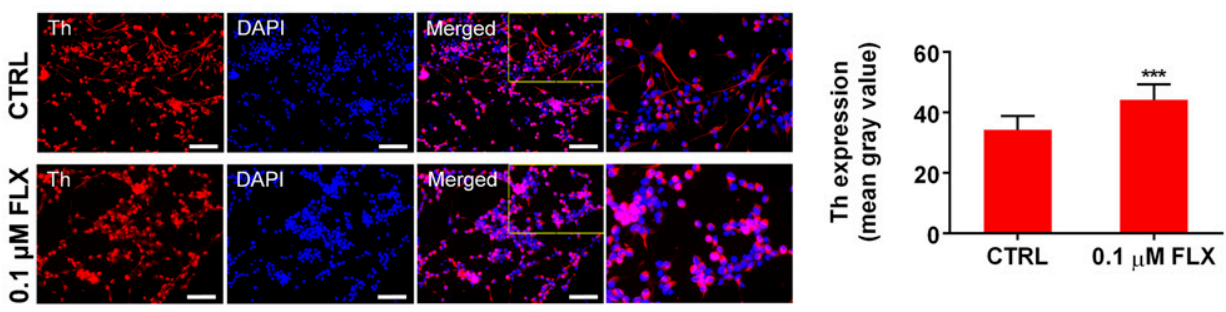

Fig. 1. Dopaminergic marker expression in mDPCs. (A) Th and Slc6a3 mRNA expression relative to Rplp0 expression in mDPCs treated with solvent (CTRL) or with $0.1 \mu \mathrm{M}$ FLX for 6 days. (B) Representative images of Th immunoreactivity in mDPCs upon control (CTRL) or $0.1 \mu \mathrm{M}$ FLX treatments. Right panels represent quantifications (mean gray value). Yellow box insets in (B) representing spindleshaped (in $0.1 \mu \mathrm{M}$ FLX) and normally branched Th positive cells (in CTRL) are enlarged in right-most column. (C) Neurite length measurements of Th positive cells in control (CTRL) or $0.1 \mu \mathrm{M}$ FLX-treated mDPCs. Data represent means \pm CI from three independent experiments in triplicates. Significant differences [two-way ANOVA for (A); $t$ test for (B) and (C)] as compared with control are marked with asterisks $(* * P<0.01$ *** $P<0.001)$. Scale bar, $100 \mu \mathrm{m}$ in (B). 
A

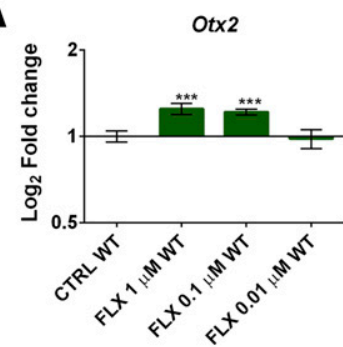

C

E

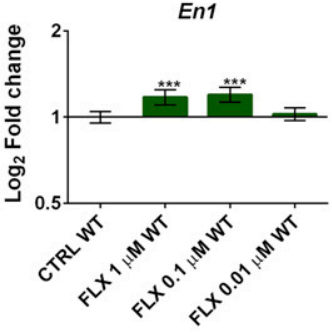

B

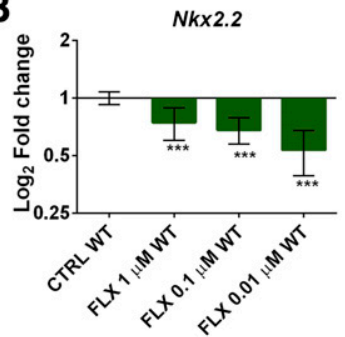

D

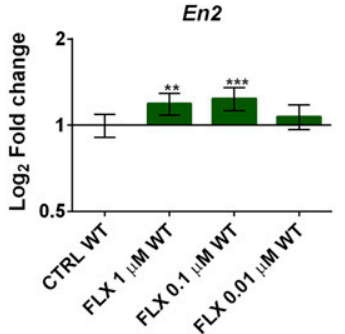

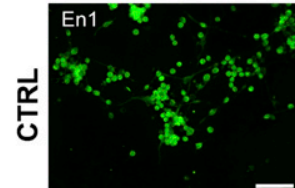
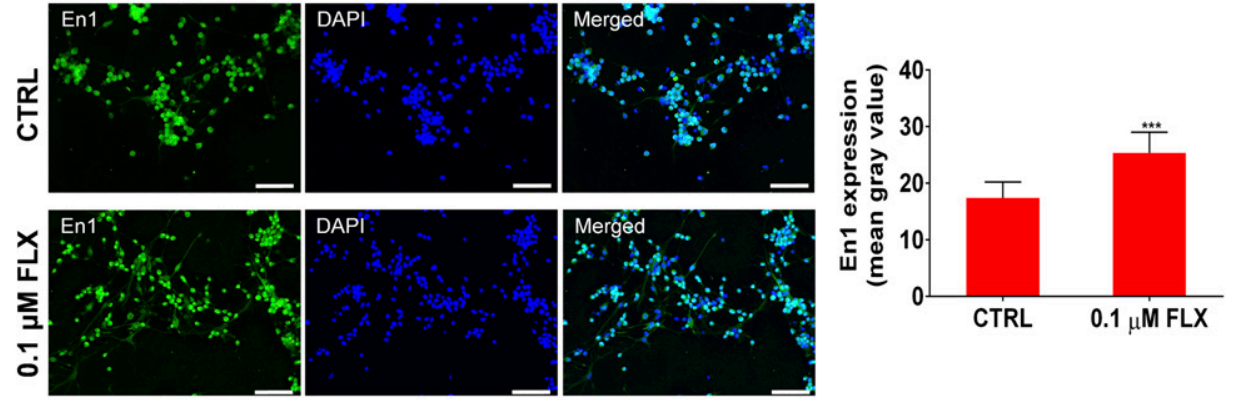

Fig. 2. FLX affects factors essential for midbrain patterning, specification, and mDA neurogenesis. WT NPCs were differentiated into mDPCs and concomitantly treated with $1,0.1$, and $0.01 \mu \mathrm{M}$ FLX Subsequently, mRNA expression of Otx2 (A), $N k x 2.2$ (B), $E n 1$ (C), and $E n 2$ (D) was measured using qPCR. (E) Representative images of immunohistochemistry of En1 in control and FLX-treated midbrain precursors. Right panel represents quantification (mean gray value) of (E). (F) Expression of Pitx3 and (G) Lmx1a upon control FLX treatments. Data represent means \pm confidence interval (CI) from three independent experiments in triplicates. Significant differences [one-way ANOVA, (A)-(D, F, G), and $t$ test, (E)] compared with control are marked with asterisks $(* * P<0.01 ; * * * P<0.001)$. Scale bar, $100 \mu \mathrm{m}$ in (E).
$\mathbf{F}$

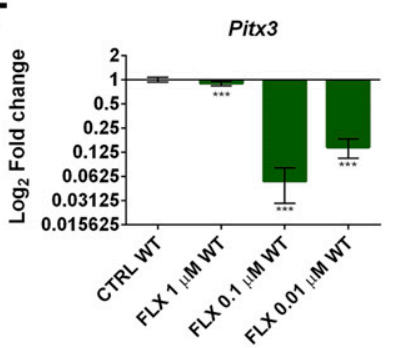

G

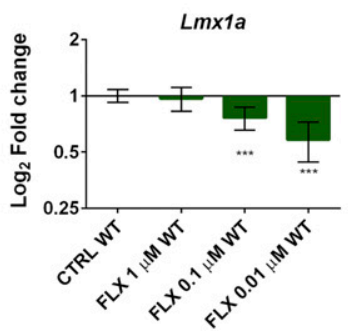

reward response and cognitive, language, and emotion processing, all of which are deficient in autistic patients (Park et al., 2016). ASD is also linked albeit partially to hereditary components (about 50\%) (Hallmayer et al., 2011), suggesting that environmental factors and toxicant exposure may play a more crucial role than initially considered (Varshney and Nalvarte, 2017).

The social motivation hypothesis of autism was developed after observations of decreased motivation to attend to social stimuli in autistic children (Scott-Van Zeeland et al., 2010). This implies dysfunctions in the brain's reward circuitry, which, in turn, has been linked to dysfunctions in dopaminergic (DA) signaling (Scott-Van Zeeland et al., 2010; Dichter et al., 2012; Pavăl, 2017). In mammals, the two main clusters of neurons that secrete dopamine are located in the midbrain and project to the nucleus accumbens, the prefrontal cortex, and the caudate putamen (Dichter et al., 2012). The development of midbrain DA (mDA) neurons is orchestrated through the activity of transcription factors and morphogens that regulate midbrain patterning and specification of midbrain progenitor cells (Arenas et al., 2015).

To date there are no studies addressing the effects of prenatal FLX exposure on the mDA differentiation process as a possible source of abnormal DA neurotransmission during development. As mentioned above, mDA neurons are the main source of DA projections in the mammalian brain, and their early differentiation, in addition to maturation, could be critical for normal DA neurotransmission in the mesocorticolimbic and nigrostriatal pathways.

Mouse embryonic stem cells (mESC) offer a reliable and validated in vitro model for the assessment of prenatal toxicity of chemicals, including drugs (Bremer and Hartung, 2004). Recently, a well-defined mESC neural differentiation model (Lee et al., 2000) was successfully used to generate mDA neurons from mESCs in vitro, a process that shown to be, to a certain extent, dependent on $\operatorname{ER} \beta$ expression (Varshney et al., 2017). For the present study, mDA neural precursors generated from mESCs were used to assess the 
A

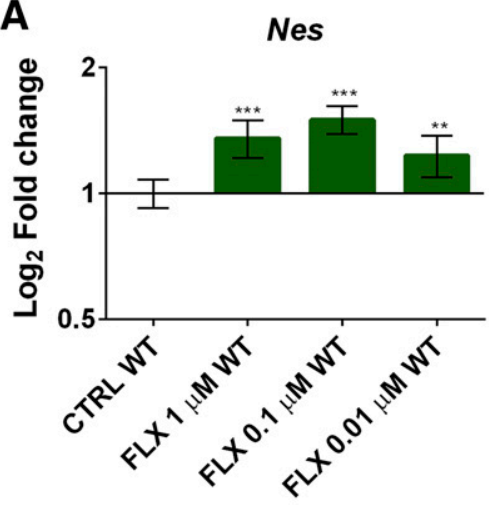

C

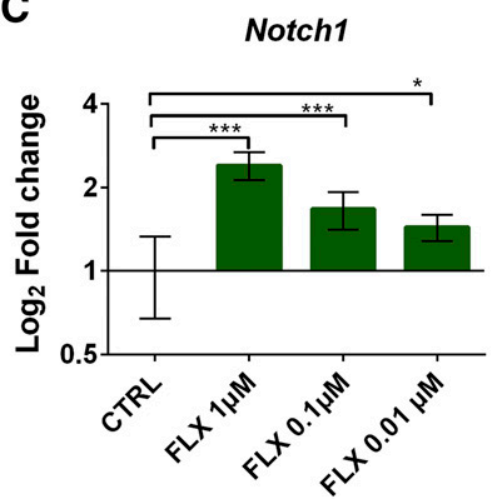

B

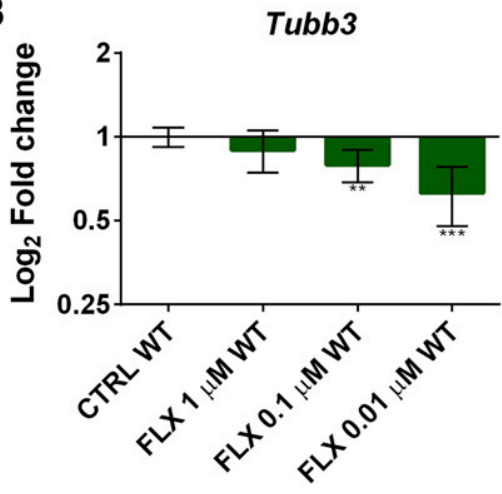

D

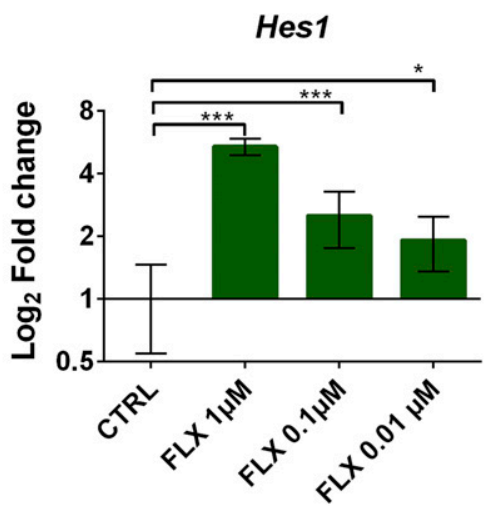

Fig. 3. FLX affects factors of neural stemness and differentiation markers in mDPCs. WT NPCs were differentiated into mDPCs and concomitantly treated with $1,0.1$, and $0.01 \mu \mathrm{M}$ FLX. Subsequently, mRNA expression of Nes (A), Tubb3 (B), Notch1 (C), and Hes1 (D) was measured using qPCR. Data represent means \pm CI from three independent experiments in triplicates. Significant differences (one-way ANOVA) compared with control are marked with asterisks $\left(* P<0.05 ;{ }^{* *} P<0.01\right.$; $* * * P<0.001)$ effect of therapeutically relevant doses of FLX on the expression of key factors involved in midbrain fate specification. As this cell model lacks serotonergic synapses and considering previous evidence that FLX can affect estrogen receptor mRNA levels and transcriptional activity (Foran et al., 2004; Mennigen et al., 2008, 2010; Lister et al., 2009; Schultz et al., 2011; Pop et al., 2015), we also addressed the involvement of ERs as a potential mode of action in FLX's effects.

\section{Materials and Methods}

Culture and Maintenance of Neural Progenitor Cells. Wildtype (WT) and ER $\beta$-knockout (BERKO) neural precursor cells (NPCs) were derived from mESCs as described earlier (Lee et al., 2000; Varshney et al., 2017). NPCs were grown in neural expansion media containing KnockOut DMEM/F-12 (without glutamine and HEPES; Life Technologies/Thermo Fisher Scientific, Paisley, UK) supplemented with $1 \% \mathrm{~N}-2$ Max (R\&D Systems, Abingdon, UK), 0.1\% gentamycin (from a $50 \mathrm{mg} / \mathrm{ml}$ solution; Life Technologies), $8.6 \mathrm{mM}$ glucose (Life Technologies), $0.5 \mathrm{mM}$ L-glutamine (Life Technologies), $20 \mathrm{mM} \mathrm{NaHCO}_{3}$ (Life Technologies), $10 \mathrm{ng} / \mathrm{ml} \mathrm{FGF} \mathrm{basic} \mathrm{(PeproTech,}$ Rocky Hill, NJ), and $1 \mu \mathrm{g} / \mathrm{ml}$ laminin (Novus Biologicals, Abingdon, UK). NPCs were plated on poly-L-ornithine $(50 \mu \mathrm{g} / \mathrm{ml}$; Sigma-Aldrich, Steinheim, Germany) and laminin (1 $\mu \mathrm{g} / \mathrm{ml}$; Novus Biologicals)coated dishes at a seeding density of $6 \times 10^{4}$ to $1 \times 10^{5}$ cells $/ \mathrm{cm}^{2}$. Media was changed every day and cells were passaged at confluency using Accutase (Life Technologies) for dissociation and spinning at 1500 rpm for 5 minutes.

Differentiation of NPCs to Midbrain Precursor Neurons. NPCs (WT and BERKO) were differentiated to midbrain dopaminergic precursor cell (mDPCs) by midbrain fate specification. In brief, NPCs were plated on poly-L-ornithine $(50 \mu \mathrm{g} / \mathrm{ml})$ and laminin $(1 \mu \mathrm{g} / \mathrm{ml})$-coated dishes at a seeding density of $1.5-2 \times 10^{5} \mathrm{cells} / \mathrm{cm}^{2}$ in neural expansion media supplemented with fibroblast growth factorbasic (FGFb, $10 \mathrm{ng} / \mathrm{ml}$; PeproTech), fibroblast growth factor 8 basic
(FGF-8b, 100 ng/ml; Miltenyi Biotec GmbH, Teterow, Germany), Sonic Hedgehog (Shh, $400 \mathrm{ng} / \mathrm{ml}$; PeproTech) and ascorbic acid $(200 \mu \mathrm{m}$; Tocris, Bristol, UK). The culture media was also supplemented with experimental doses of FLX at 1, 0.1 and $0.01 \mu \mathrm{M}$ (in DMSO) or DMSO at $0,1 \%$ (solvent control). Cells were maintained in this media for 6 days with a daily change of media. During this period NPCs generate midbrain precursor cells. On the 6th day media was removed and cells were washed with PBS and lysed with Buffer RLT (Qiagen, Hombrechtikon, Switzerland) supplemented with 1\% $\beta$-mercaptoethanol.

Quantitative Real-Time PCR Analysis. Total RNA was isolated from mDPCs using the AllPrep DNA/RNA Mini Kit and treated with DNase I (both from Qiagen). RNA concentrations were measured using a NanoQuant plate on Tecan Infinite M200 (Tecan, Männedorf, Switzerland), and RNA quality was checked with Bioanalyzer (Agilent Technologies, Santa Clara, CA). Two thousand nanograms of RNA was used for reverse transcription with Superscript VILO cDNA Synthesis Kit (Life Technologies). Quantitative Real-Time polymerase chain reaction (qPCR) was performed using $5 \mathrm{ng}$ of cDNA, selfdesigned (Primer 3.0 web) exon-exon spanning primers (see Table 1) and KAPA SYBR Fast Master Mix ABI Prism (Kapa Biosystems, Wilmington, MA), in a 7500 RealTime PCR System (Applied Biosystems, Foster City, CA). PCR efficiency was calculated for each primer set using standard curves with serial dilutions of test samples. Primer efficiency was 2 in most of the sets used, representing a doubling in each cycle. Threshold cycle values for targets were determined in triplicates and $\mathrm{PO}$ large ribosomal protein $\mathrm{RplpO}$ (known as 36B4) was used as a reference gene. Relative fold changes compared with WT or BERKO controls were calculated using the Pfaffl or $\Delta \Delta$ Ct method.

Immunocytochemistry and Fluorescence Imaging. Cells were grown on poly-L-ornithine and laminin-coated chambered culture slides (Ibidi, Martinsried, Germany) and fixed in ice-cold 4\% paraformaldehyde (Santa Cruz Biotechnology, Dallas, TX) for 15 minutes followed by three washes [phosphate-buffered saline (PBS), 
A

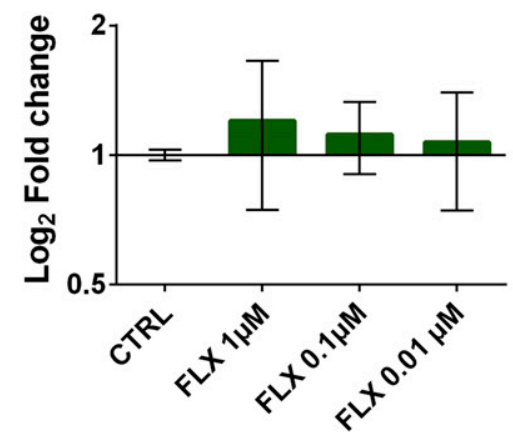

C

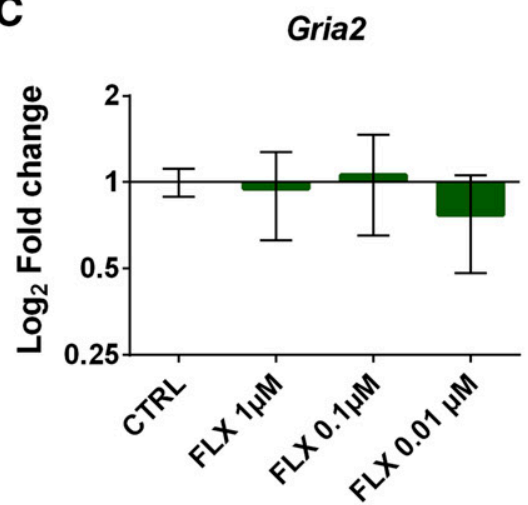

B

Pax3

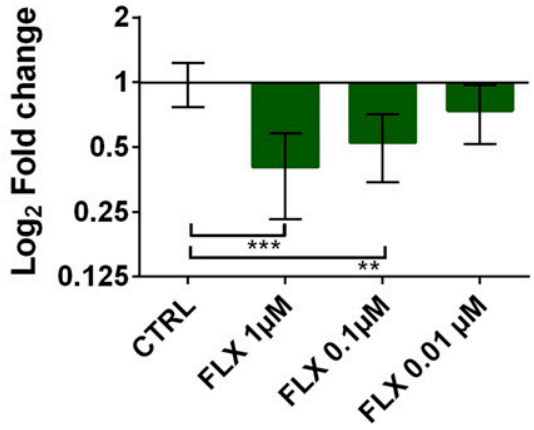

D

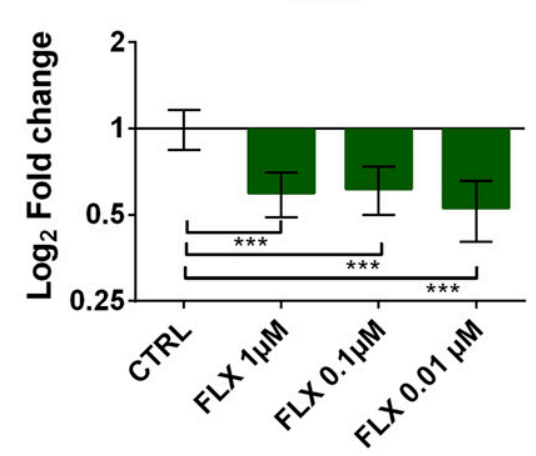

Fig. 4. FLX treatment of mDPCs affects some genes associated with autism. Relative change in mRNA expression levels of (A) Foxp2, (B) Pax3, (C) Gria2, (D) Rora, and (E) Reln for control (CTRL) or $1,0.1$, and $0.01 \mu \mathrm{M}$ FLX treatments in mDPCs. Data represent means \pm CI from three independent experiments in triplicates. Significant differences (one-way ANOVA) as compared to control are marked with asterisks (** for $P<0.01 ; * * *$ for $P<$ 0.001).

E

Reln

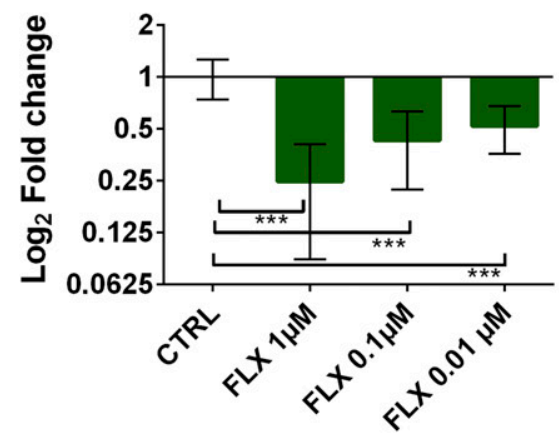

$\mathrm{pH}$ 7.4], incubated with blocking serum (1\% bovine serum albumin and $0.1 \%$ Tritonx-100; both from Sigma-Aldrich) in PBS and stained with primary antibodies against Th (R\&D Systems, Minneapolis, MN), En1 (Sigma-Aldrich), SERT (Thermo Fisher Scientific, Waltham, MA) followed by detection with Alexa Fluor secondary antibodies (Thermo Fisher Scientific) and DAPI (300 nM; Molecular Probes, Eugene, OR) with PBS washes between and after each incubation. Slides were observed under Axioplan2 epifluorescent microscope (Carl Zeiss, Oberkochen, Germany) using $20 \times$ objective (FluorPlan) with appropriate filter sets for each fluorophore and images were captured with Zeiss Axiocam MR scientific camera using Axiovision 4.0 software (Carl Zeiss) Appropriate negative and positive controls were incorporated for respective primary and secondary antibodies.

Image Analysis. Quantification of immunoexpression density was performed by analyzing images in Fiji (ImageJ; National Institutes of Health, Bethesda, MD) image analysis software, using similar settings for each marker across all images and experimental conditions. Neurite length measurements were performed on calibrated images using NeuronJ plugin in Fiji (Meijering et al., 2004) with length threshold above $5 \mu \mathrm{m}$.

In Silico ER Binding Predictions. A molecular docking approach was used to detect potential interactions between FLX and the ERs. The X-ray structures of ER $\alpha$ and ER $\beta$ (Protein Data Bank ID: 1sj0 and $2 \mathrm{fsz}$ respectively) were acquired from the "A Database of Useful Decoys: Enhanced" [http://dude.docking.org/] database (Mysinger et al., 2012). A dataset of ER ligands containing 131 active and 101 inactive compounds was obtained from the U.S. Food and Drug Administration's Endocrine Disruptor Knowledge Base (EDKB) (Ding et al., 2010). The protein structures were prepared for docking using the Schrödinger Protein Preparation Wizard and the compounds from the EDKB ER dataset were prepared using the default settings of Schrödinger LigPrep. Docking simulations were performed using Schrödinger Glide (Friesner et al., 2004), with the default settings of the Glide SP (Single Precision) tool. The docking scores were then subsequently binned into 0.5 bins. 
Statistical Analysis. Experiments were performed three times with three technical replicates. If not stated otherwise, the statistical differences between groups were analyzed using the GraphPad Prism 6 software by one-way analysis of variance (ANOVA), followed by Dunnett's test for multiple comparisons, or by Student's $t$ test for single comparisons. $P$ values $<0.05$ were considered statistically significant unless stated otherwise. Data are shown as means \pm $95 \%$ confidence intervals.

\section{Results}

FLX Affects the Differentiation of NPCs to Midbrain Precursors. To assess if FLX affects early differentiation of the midbrain, we used a well-defined mESC model that mimics neural development in vitro, which is marked by the presence of early generating neural precursor cells (NPCs) and subsequent induction of midbrain dopaminergic precursor cells (mDPCs). Although immature, these mDPC cultures contain dopaminergic neuronal precursors that express tyrosine hydroxylase $(T h)$ as well as low levels of the dopamine transporter (Slc6a3) (Fig. 1A). However, these cells are nascent and do not form synapses yet. Using this cell model, we analyzed the expression of genes that are critical for the early development of $\mathrm{mDPCs}$, as well as $\mathrm{mDA}$ neurogenesis, following treatment with therapeutically relevant FLX concentrations. NPCs were differentiated to mDPCs by midbrain fate specification using a combination of FGFb, FGF-8b, Shh, and ascorbic acid. The cells were maintained for 6 days in culture medium with the induction cocktail and concomitantly exposed to a therapeutically relevant dose of $\mathrm{FLX}(0.1 \mu \mathrm{M})$ or $0.1 \%$ DMSO (solvent control). Expression of Th was confirmed by immunocytochemistry (Fig. 1B) in control and FLX-treated cells and was higher in the FLX-treated group $(44.13 \pm 1.216)$ compared with control $(34.28 \pm 1.302)$ (Fig. 1A). However, the neuronal processes of Th + cells in the FLX-treated group were smaller [mean neurite length $(\mu \mathrm{m}) 11.72 \pm 0.5814$ ] (Fig. 1C) and spindle shaped compared with well branched and longer processes in control group (19.37 \pm 0.6112$)$ (Fig. 1B, magnified region of interest), suggesting that FLX interferes with the differentiation process of dopaminergic cells.

To investigate underlying transcriptional changes, mDPCs were treated with FLX $(1,0.1$, or $0.01 \mu \mathrm{M})$ and expression of genes critical for early regional specification and patterning of the ventral midbrain were measured. First, we analyzed the expression of the transcription factors orthodenticle homeobox 2 (Otx2) and homeobox engrailed-1 and 2 (En1 and En2). FLX treatment at 1 and $0.1 \mu \mathrm{M}$ increased mRNA expression of Otx2 (1.24 and 1.21-mean-fold change, respectively, $P<0.001$, Fig. 2A), of En1 (1.17- and 1.19-fold change, respectively, $P<0.001$, Fig. 2C) and of $E n 2$ (1.18-fold change, $P=0.015$ and 1.23 -fold change, $P=0.0003$, Fig. $2 D)$. In contrast, the expression of NK2 homeobox 2 (Nkx2.2), involved in midbrain fate determination and negatively regulated by $\operatorname{Otx} 2$, was downregulated by FLX treatment at all tested concentrations $(P<0.0001$, Fig. 2B). Immunocytochemistry for En1 confirmed its higher expression in mDPCs following FLX treatment $(25.34 \pm 0.8686)$ compared with solvent control (17.40 $\pm 0.8201, n=12$ ) (Fig. $2 \mathrm{E})$.

We further analyzed the expression of transcription factors essential for mDA neurogenesis, i.e., paired-like homeodomain transcription factor 3 (Pitx3) and LIM homeobox transcription factor $1 \alpha(\operatorname{Lmx} 1 a)$. FLX treatment at all concentrations, except $1 \mu \mathrm{M}$ FLX for Lmx1a, downregulated expression of both Pitx3

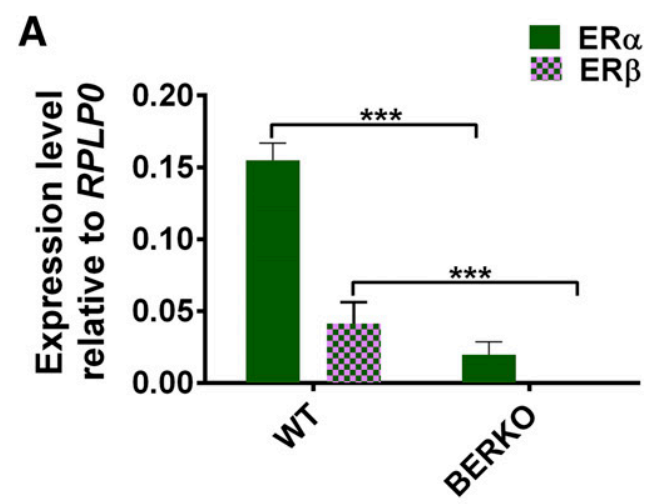

$\mathbf{B}$

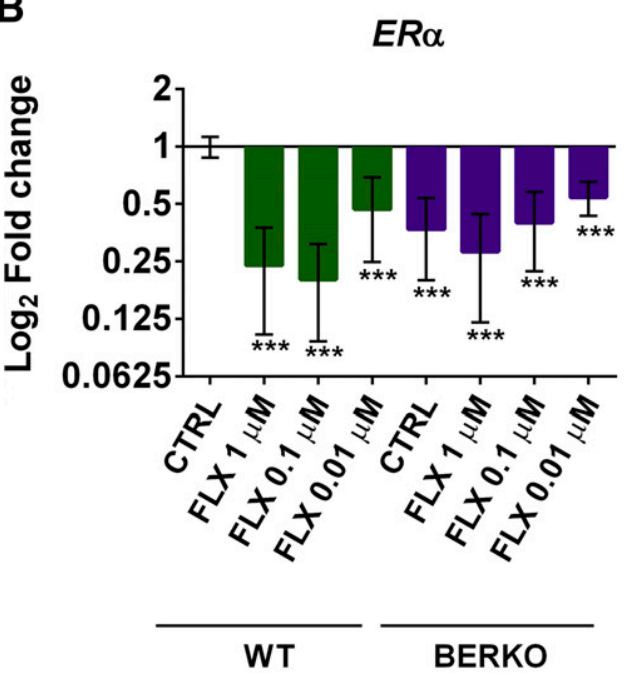

C
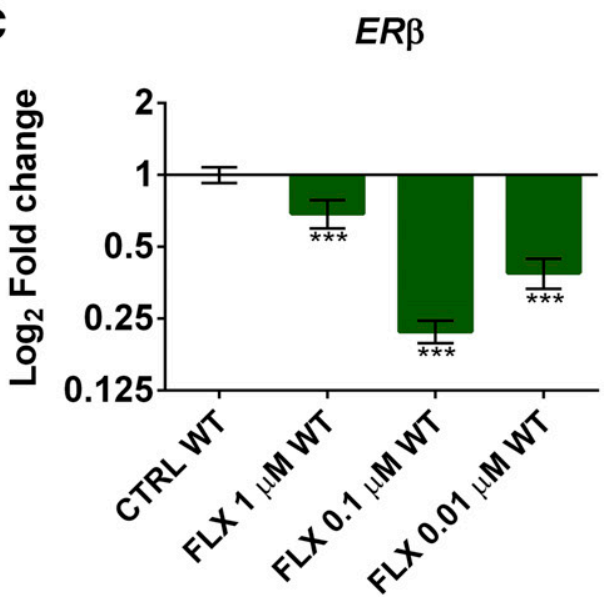

Fig. 5. FLX affects $\mathrm{ER} \alpha$ and $\mathrm{ER} \beta$ expression in mDPCs. WT and BERKO NPCs were differentiated into mDPCs and concomitantly treated with 1, 0.1, and $0.01 \mu \mathrm{M}$ FLX. (A) mRNA expression of $E R \alpha$ and $E R \beta$ relative to $R p l p 0$ in WT and BERKO mDPCs, (B) fold change in mRNA expression of $E R \alpha$ in WT and BERKO mDPCs and (C) $E R \beta$ in WT mDPCs following FLX treatments. Data represent means \pm CI from three independent experiments in triplicates. Significant differences (one-way ANOVA) compared with WT CTRL are marked with asterisks $(* * * P<0.001)$.

(0.89-fold change by $1 \mu \mathrm{M}$ FLX $P=0.0006 ; 0.05$-fold change by $0.1 \mu \mathrm{M}$ FLX, $P<0.001$, and 0.14 -fold change by $0.01 \mu \mathrm{M}$ FLX, $P<0.001$ respectively) and $L m x 1 a(0.76$-fold change by $0.1 \mu \mathrm{M}$ FLX, $P=0.0008$ and 0.58 -fold change by $0.01 \mu \mathrm{M}$ FLX, $P<0.001$ ) (Fig. 2G). 
A
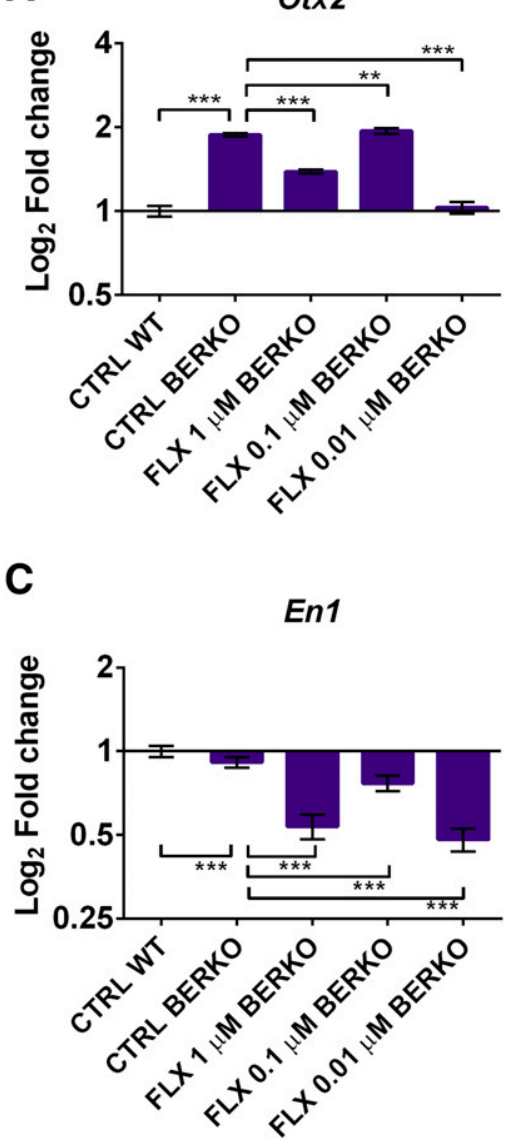

B

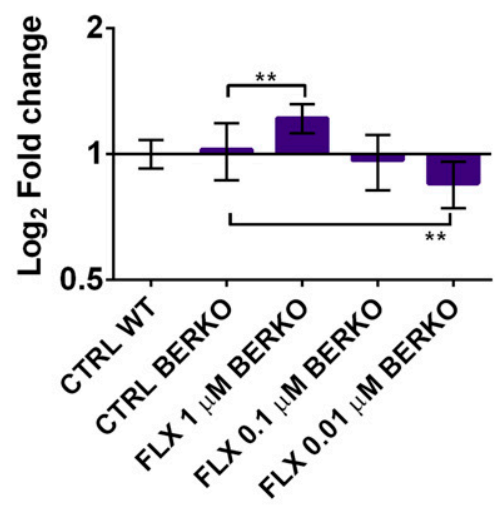

D

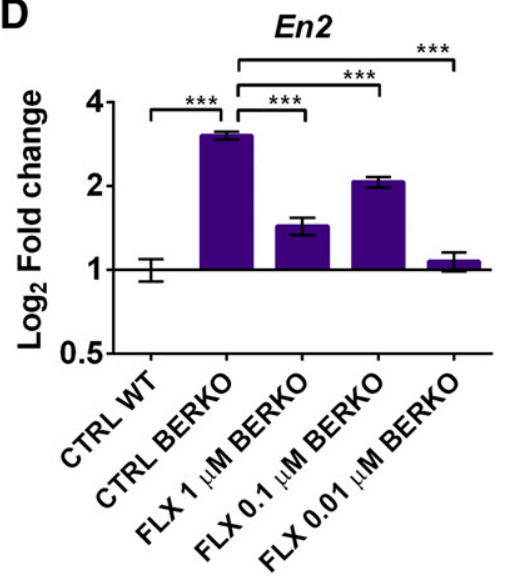

Fig. 6. Loss of $\mathrm{ER} \beta$ attenuates the FLX-mediated effects on factors essential for midbrain patterning and $\mathrm{mDPC}$ specification. BERKO NPCs were differentiated into mDPCs and concomitantly treated with $1,0.1$, and 0.01 $\mu \mathrm{M}$ FLX. Subsequently, mRNA expression of Otx2 (A), $N k x 2.2$ (B), En1 (C), and En2 (D) was measured using qPCR. Data represent means \pm CI from three independent experiments in triplicates. Significant differences (one-way ANOVA) are marked with asterisks $\left({ }^{* *} P<0.01\right.$; $* * * P<0.001)$.
Next, we assessed whether FLX affects stemness by analyzing the expression of the neural stem cell marker Nestin (Nes) and the neuronal marker $\beta 3$-tubulin (Tubb3) in mDPCs. We found that Nes expression was increased (Fig. 3A) by FLX ( $1 \mu \mathrm{M}$ FLX caused a 1.35-fold change, $P<0.001$; FLX $0.1 \mu \mathrm{M}$ caused a 1.50 -fold change, $P<0.001$ and FLX $0.01 \mu \mathrm{M}$ caused a 1.23-fold change, $P=0.0010$ ) and Tubb3 (Fig. 3B) was decreased by lower concentrations of FLX $(0.1 \mu \mathrm{M}$ FLX induced a 0.79 -fold change, $P=0.0028$ and $0.01 \mu \mathrm{M}$ FLX induced a 0.63 -fold change, $P<$ 0.0001). Proper Notch-Hes signaling is essential for maintaining the neural progenitor identity and suppression of neuronal differentiation (Schuurmans and Guillemot, 2002). By analyzing the expression of Notch1 and its downstream effector Hes1, we observed a marked increase in the expression of both genes upon FLX treatment (Notch1: 2.40-fold change by $1 \mu \mathrm{M}$ FLX $P<$ 0.0001 ; 1.67 -fold change by $0.1 \mu \mathrm{M}$ FLX,$P<0.0001$; and 1.43 -fold change by $0.01 \mu \mathrm{M}$ FLX, $P<0.05$. Hes1: 5.38 -fold change by $1 \mu \mathrm{M}$ FLX $P<0.0001 ; 2.51$-fold change by $0.1 \mu \mathrm{M}$ FLX, $P<0.001$; and 1.91-fold change by $0.01 \mu \mathrm{M}$ FLX, $P<0.05$.) (Fig. 3, C and D). These data suggest that FLX interferes with signaling cascades underlying precursor cell maintenance and maturation.

FLX Affects Expression of Genes Associated with Autism. To evaluate if FLX modulates the expression of genes associated with ASD we measured the expression of forkhead box protein P2 (Foxp2), paired box 3 (Pax3), glutamate ionotropic receptor AMPA type subunit 2 (Gria2), retinoic acid receptorrelated orphan receptor- $\alpha$ (Rora), and reelin (Reln), each of which not only plays a crucial role in midbrain patterning and neurogenesis (Nguyen et al., 2010; Sarachana et al., 2011; Lammert and Howell, 2016) but has also been implicated in autism (Borg et al., 2002; Nguyen et al., 2010; Bowers and Konopka, 2012; Uzunova et al., 2014; Lammert and Howell, 2016). We observed a downregulation of the expression of Pax3 (0.41-fold change by $1 \mu \mathrm{M}$ FLX, 0.52 -fold change by $0.1 \mu \mathrm{M}$ FLX, $P<0.001$ ), Rora ( 0.60 -fold change by $1 \mu \mathrm{M}$ FLX, $P<0.05$; 0.61 -fold change by $0.1 \mu \mathrm{M}$ FLX, $P<0.05$; and 0.53 -fold change by $0.01 \mu \mathrm{M}$ FLX, $P<0.01)$, and Reln $(0.25$-fold change by $1 \mu \mathrm{M}$ FLX, $P<0.001$; 0.43 -fold change by $0.1 \mu \mathrm{M}$ FLX, $P<0.0001$; and 0.52 -fold change by $0.01 \mu \mathrm{M}$ FLX, $P<0.01$ ) (Fig. 4). Additionally, increased levels of En1 and En2, as shown in Fig. 2 , are also reported to correlate with ASD (Kuemerle et al., 2007; James et al., 2014). These data suggest that FLX may indeed influence the expression of genes associated with ASD.

FLX Affects ER Expression in Midbrain Precursors. Since the mDPC cultures are too immature to form functioning synapses and any 5-HT produced is heavily diluted in the culture medium, the effect of FLX on the genes analyzed in this study is probably not due to 5-HT reuptake inhibition. Increasing evidence points to FLX having estrogen-disrupting effects (Foran et al., 2004; Mennigen et al., 2008, 2010; Lister et al., 2009; Schultz et al., 2011; Lupu et al., 2017), and in view of our recent data showing that $\mathrm{ER} \beta$ plays an important role in the differentiation of midbrain dopaminergic neurons in vitro (by maintaining proper Notch-Hes signaling, among others) (Varshney et al., 2017), we investigated by using $\mathrm{ER} \beta$ knockout (BERKO) cells whether treatment with this SSRI affects the expression levels of ERs in WT mDPCs, as well as whether $\operatorname{ER} \beta$ is involved in the observed effects of FLX. 
A

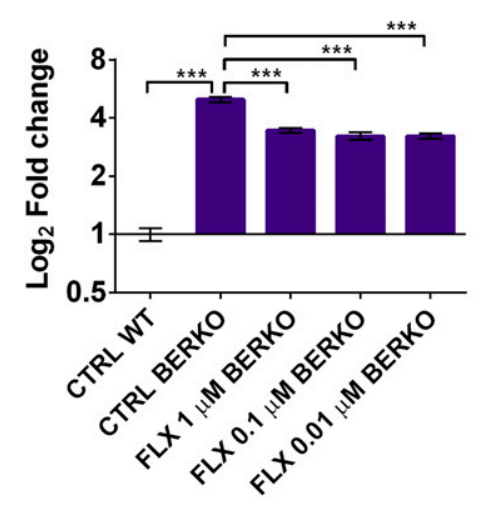

C

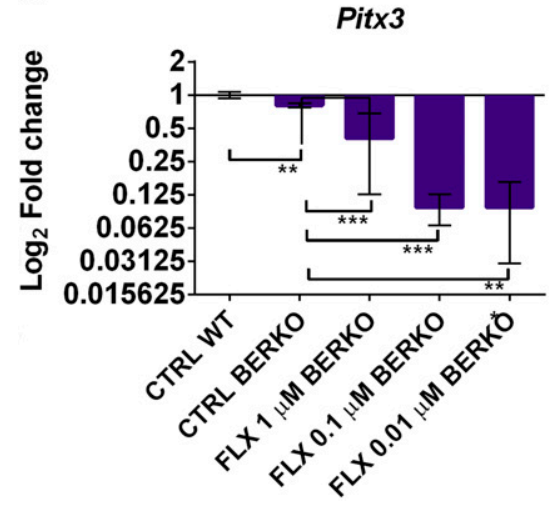

B

Tubb3

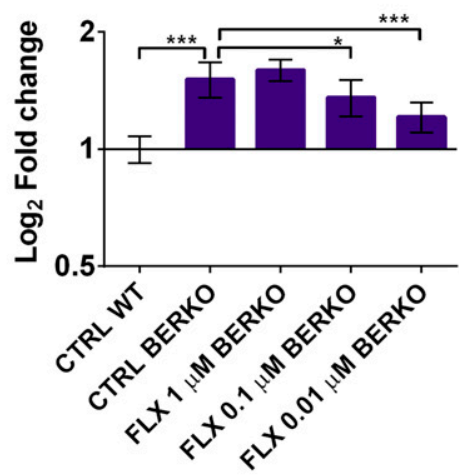

D

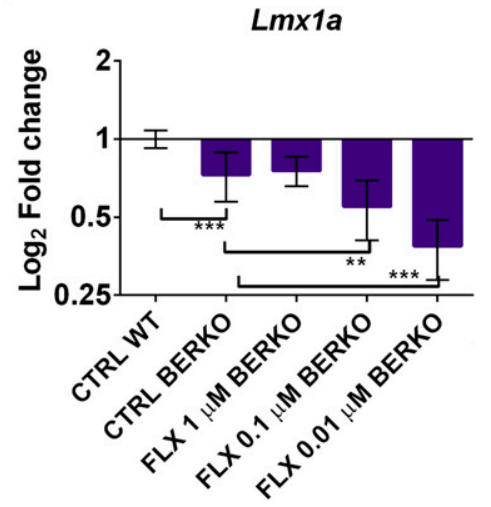

Fig. 7. FLX affects factors of neural stemness and $\mathrm{mDA}$ neurogenesis in mDPCs. BERKO NPCs were differentiated into mDPCs and concomitantly treated with $1,0.1$, and 0.01 $\mu \mathrm{M}$ FLX. Subsequently, mRNA expression of (A) Nes, (B) Tubb3, (C) Pitx3, and (D) Lmx1a was measured using qPCR. Data represent means \pm CI from three independent experiments in triplicates. Significant differences (one-way ANOVA) are marked with asterisks $\left(* P<0.05\right.$, ${ }^{* *} P<0.01$; $* * * P<0.001)$.
We observed that both $\operatorname{ER} \alpha$ and $\operatorname{ER} \beta$ are expressed in mDPCs (Fig. 5A) and that ER $\alpha$ mRNA expression was downregulated in both BERKO mDPCs $(0.13$-fold, $P<0.001)$ and in WT mDPCs after FLX treatment compared with solvent control (0.24-, 0.20-, and 0.47-fold change by $1,0.1$, and $0.01 \mu \mathrm{M}$ FLX, respectively, $P<0.001$, Fig. 5B). Interestingly, in BERKO mDPCs, ER $\alpha$ levels were lower than in WT cells and FLX treatment did not reduce $\mathrm{ER} \alpha$ expression any further compared with treatments in WT cells (Fig. 5B). ER $\beta$ mRNA expression was also downregulated in WT mDPCs after FLX treatment compared with solvent control (0.69, 0.22-, and 0.39 -fold change by $1,0.1$ and $0.01 \mu \mathrm{M}$ FLX, respectively, $P<0.001$, Fig. 5C).

FLX Effects on Midbrain Fate Specification, But Not Maturation, Are Dependent on ER $\boldsymbol{\beta}$. To assess the role of ERs in mediating the effects of FLX on DA differentiation, we used BERKO NPCs to measure gene expression of the key factors analyzed above. We observed that in BERKO mDPCs, FLX had no or even opposite effects on the genes involved in mDPC specification (Otx2, Nkx2.2, En1, and En2) (Fig. 6) and on the stemness marker Nes (Fig. 7A), suggesting that ERs are involved in the effects of FLX on early midbrain fate specification. In BERKO cells, Otx2, En1, En2, and Nes mRNA expression was downregulated after FLX treatment, compared with BERKO solvent controls (Fig. 6, A, C, and D; Fig. 7A). Likewise, FLX treatment of BERKO mDPCs did not affect expression of the autism-related genes Rora or Reln, which was 3-fold and 15-fold decreased, respectively, in BERKO compared with WT mDPCs in control-treated cells (Fig. 8).
On the other hand, in BERKO mDPCs FLX induced effects similar to those observed in WT mDPCs on the neuronal marker Tubb3 (0.90-fold change by $0.1 \mu \mathrm{M}$ FLX, $P=0.0104$; 0.80 -fold change by $0.01 \mu \mathrm{M}$ FLX, $P<0.0001$, Fig. 7B) and on the neurogenesis factors Pitx3 (0.50-, 0.12-, and 0.12-fold change by 1, 0.1, and $0.01 \mu \mathrm{M}$ FLX, respectively, $P<0.001)$ and $L m x 1 a$ (0.75-fold change, $P=0.001$ for FLX $0.1 \mu \mathrm{M}$; 0.53-fold change, $P<0.001$ for FLX $0.01 \mu \mathrm{M})$, decreasing their mRNA expression compared with BERKO solvent controls (Fig. 7, C and D).

FLX Is a Potential ER Binder. As our results indicate the involvement of ERs in mediating some of the effects of FLX, we investigated if FLX could bind these receptors using molecular docking predictions. The results showed that both $\operatorname{ER} \alpha$ and $\operatorname{ER} \beta$ can bind FLX through a combination of hydrophobic and polar interactions (Fig. 9). Hydrophobic interactions within ER ligand binding pockets were mainly mediated by leucine/isoleucine (Leu/Ile) and phenylalanine (Phe) amino acid residues, whereas hydrogen bonds could form between the secondary amino group present in FLX and glutamate (Glu353/Glu42) residues in ER ligand binding pockets. The low docking energies, located in the -9.0 to -8.5 bin for both $\operatorname{ER} \alpha$ and $\operatorname{ER} \beta$ (Fig. 10), suggest that FLX has a fairly strong tendency to bind to ERs. The probabilities for binding of FLX to $\operatorname{ER} \alpha$ and $\operatorname{ER} \beta$ are 0.88 and 0.74 , respectively.

\section{Discussion}

To address prenatal FLX effects on the early differentiation of $\mathrm{mDA}$ fate from neural precursors, we employed a well 


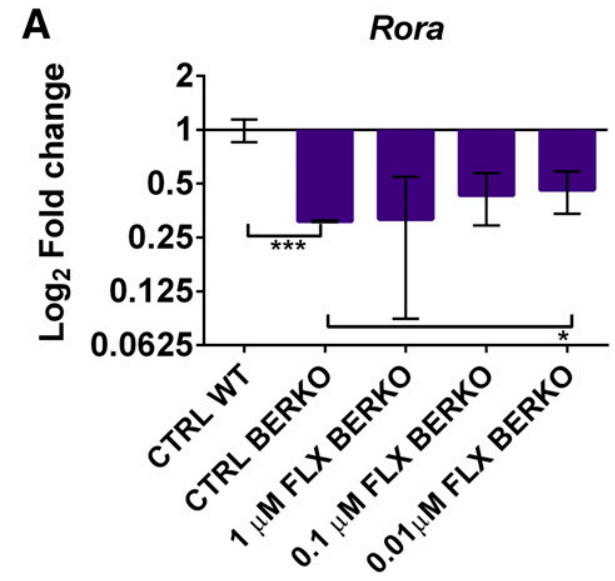

B

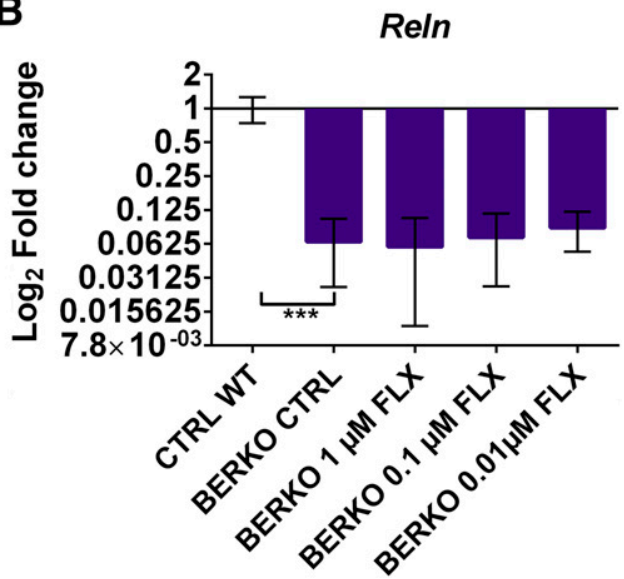

Fig. 8. FLX affects ASD-associated genes important for neuronal development. WT and BERKO NPCs were differentiated into mDPCs and concomitantly treated with $1,0.1$, and $0.01 \mu \mathrm{M}$ FLX. Subsequently, mRNA expression of Rora (A) and Reln (B) was measured using qPCR. Data represent means \pm CI from three independent experiments in triplicates. Significant differences (two-way ANOVA, followed by Turkey's test) are marked with asterisks $(* P<0.05 ; * * * P<0.001 ; \mathrm{ns}$, no significance).

defined and highly controlled mESC cell model that reflects many of the key aspects of midbrain stem cell fate acquisition and development (Lee et al., 2000; Varshney et al., 2017). In vivo, the development of midbrain dopaminergic neurons begins with the patterning of the neural tube and is orchestrated by the isthmic organizer and the floor plate (Arenas et al., 2015). The isthmic organizer is located at the midbrain-hindbrain boundary and is represented by a group of cells with a complex pattern of gene expression that includes the signaling factors FGF8 (fibroblast growth factor 8) and Wnt1 (wingless-int1) and transcription factors Otx2, Gbx2 (gastrulation brain homeobox 2), Pax2 (paired box 2), and En1/2, among others (Arenas et al., 2015). The midbrain floor plate is a glial structure located along the ventral midline of the neural tube and modulates cell identity in the dorsalventral position by secreting the morphogens Shh and Wnt1 (Arenas et al., 2015). Thus, the coordinated activity of these transcription factors and morphogens regulates ventral midbrain patterning and the specification of ventral midbrain progenitor cells. Besides ventralization, the midbrain floor plate has neurogenic potential and is the source of dopaminergic precursors, subsequently giving rise to dopaminergic neurons (Hegarty et al., 2013). In vitro, mature midbrain dopaminergic neurons ( $\mathrm{mDNs}$ ) have been successfully generated from mESCs, using highly controlled culture conditions to guide the differentiation process toward NPCs and, subsequently, to mDPCs and mDNs (Varshney et al., 2017). The $\mathrm{mDNs}$ obtained from both $\mathrm{WT}$ and BERKO mDPCs express the dopaminergic markers Th (tyrosine hydroxylase, the enzyme which catalyzes the rate-limiting step of cathecolamine synthesis) and Slc6a3 (solute carrier family 6 member 3, the presynaptic dopamine transporter) and have functional dopamine synthesis and release (Varshney et al., 2017).

Our results show that FLX exposure, in therapeutically relevant doses, affects the differentiation process from NPCs to mDPCs. To our knowledge, there are no data on FLX kinetics in the fetus and FLX levels in the fetal brain. Thus we considered FLX concentrations as therapeutically relevant if they correspond to infant cord blood and plasma concentrations reported in the literature, which are in the range of 0.037-1.06 $\mu \mathrm{M}$ (Heikkinen et al., 2003; Kim et al., 2006; Gurnot et al., 2015).

We found that FLX upregulates transcription factors Otx2, $E n 1$, and $E n 2$, which are essential for ventral midbrain patterning, whereas $N k x 2.2$ was downregulated. Otx2, En1, and En2, among others, are expressed as early as embryonic days 7 to 8 (E7-8) in the mouse and are critical for the formation and positioning of the isthmic organizer, thus contributing to the patterning and determination of midbrain precursors (Hegarty et al., 2013; Arenas et al., 2015). Otx2 is also essential for the neurogenic potential of the floor plate (Hegarty et al., 2013). Conversely, Nkx2.2 is a negative regulator of midbrain dopaminergic development and is repressed by Otx2 (Abeliovich and Hammond, 2007). A lower expression of $N k x 2.2$ favors dopaminergic neuron differentiation at the expense of serotonergic neurons (Abeliovich and Hammond, 2007). Thus our results suggest that FLX increases differentiation of NPCs to midbrain precursors, which might lead to a decrease in serotonergic neurons.

On the other hand, we observed that the expression of the mDA markers $L m x 1 a$ and Pitx 3 were downregulated upon FLX treatment. $L m x 1 a$ is induced by $O t x 2$ and is required for the specification of mDA neurons and suppression of alternative fates (Hegarty et al., 2013; Arenas et al., 2015). Pitx3 is part of a network of transcription factors that cooperate to induce mDA neurogenesis and is also important for $\mathrm{mDA}$ neuronal survival (Hegarty et al., 2013). Concomitantly, the stemness marker Nes was upregulated, whereas the neuronal differentiation marker Tubb3 was downregulated. Interestingly, the mRNA expression of Pitx 3, Lmx $1 a$, and Tubb3 was lower after treatment with submicromolar FLX, suggesting that at $1 \mu \mathrm{M}$, FLX may be interacting with targets that compensate the downregulation of these neurogenesis markers. Th expression was increased following submicromolar FLX $(0.1 \mu \mathrm{M})$; however, the nascent Th positive cells in this group had spindle-shaped morphology compared with more branched processes in untreated mDPCs. This could be attributable to feedback mechanisms during which cells produce more enzyme to synthesize dopamine, whereas the progenitors still cannot mature owing to aberrant signaling and expression of other factors such as reelin. Together, these findings suggest that although FLX promotes mDA fate determination, it prevents the midbrain precursors to progress into mature DA neurons.

These effects on dopaminergic differentiation might contribute to the association between developmental FLX 
A
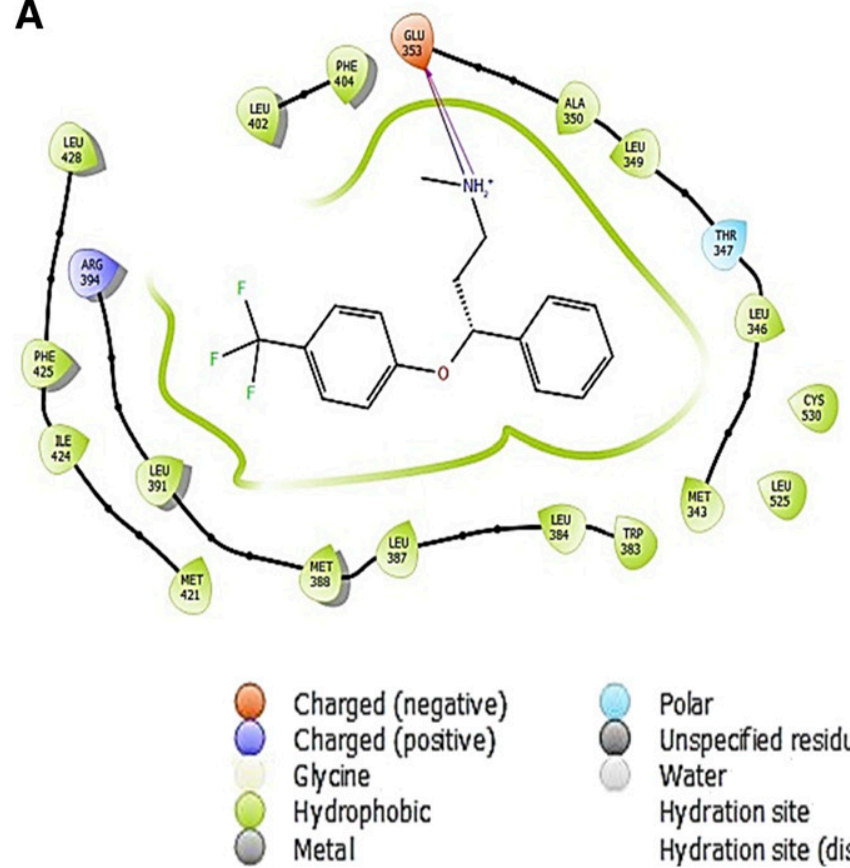
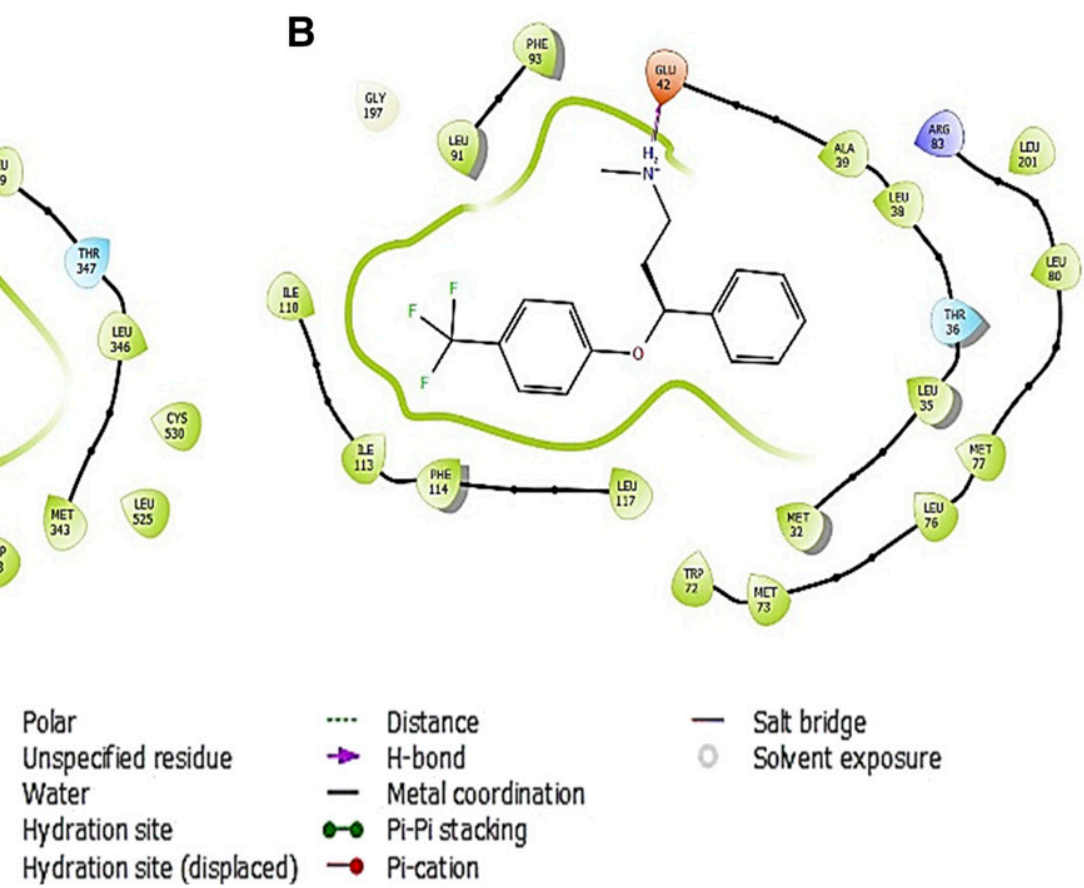

exposure and increased risk for ASD. Importantly, some of the genes that we have found dysregulated by FLX are known to be associated with ASD (En1/2, Pax3, Rora, and Reln). En2 has been associated with ASD in genetic linkage studies, and increased En2 gene expression and protein levels were detected in the cerebellum of autistic subjects (Kuemerle et al., 2007; James et al., 2014). A study of postmortem brain tissue reveled decreased Rora protein levels in the cerebellum and frontal cortex of autistic individuals, which was consistent with increased $\mathrm{CpG}$ methylation in the upstream promoter region of Rora (Nguyen et al., 2010). Likewise, decreased Reln mRNA and protein was observed in the cerebellum and superior frontal cortex of autistic subjects (Lammert and Howell, 2016). Reln is critical for proper cell positioning and neuronal migration during development, and increasing evidence suggests that heterozygous mutations in Reln, including de novo mutations, contribute to the autistic phenotype (Lammert and Howell, 2016). Finally, a cryptic deletion including part of the Pax3 gene and a de novo translocation were observed in an ASD patient (Borg et al., 2002).

Several studies suggest that FLX affects estrogenic signaling in fish and mammals (Foran et al., 2004; Mennigen et al., 2008, 2010; Lister et al., 2009; Schultz et al., 2011; Lupu et al., 2017) and FLX has been shown to modulate ER transcriptional activation at (sub)micromolar concentrations using a reporter gene assay in breast cancer cells expressing both ERs (Pop et al., 2015). Proper estrogenic signaling is essential for normal brain development and influences apoptosis, synaptogenesis, and neuronal morphometry (McCarthy, 2008). In a recent study, we have shown that ER $\beta$ facilitates midbrain dopaminergic fate and function largely ligand independently (Varshney et al., 2017) and that loss of ER $\beta$ had similar effects on DA differentiation, as we observe here with FLX treatment. Furthermore, ligand-mediated effects have also been shown as
NPCs treated with estradiol during differentiation to $\mathrm{mDPCs}$ in vitro resulted in an increased proliferation of mDPCs expressing $L m x 1 a$ and in a higher proportion of dopaminergic neurons (Díaz et al., 2009). Estradiol action was mediated through nuclear ERs, as ICI182780 (ER antagonist) abolished the effect on proliferation of committed precursors and subsequent differentiation (Díaz et al., 2009). In our study, we showed that FLX decreases ER $\alpha$ and $\mathrm{ER} \beta$ levels and that its effects on dopaminergic differentiation are abolished or even counteracted in BERKO cells, with the exception of Tubb3, Lmx1a, and Pitx3 downregulation. Notably, ER $\alpha$ is also strongly decreased in the BERKO cells and we show that FLX is predicted to bind to both ERs in silico, suggesting an involvement of one or both of these receptors in the observed effects. Nevertheless mDPCs do not form synapses, and although they express the serotonin transporter Slc6a4 (Supplementary Fig. 1, A and B), only low Tph2 levels are detected in these cells (Supplementary Fig. 1A), and any 5-HT produced is greatly diluted in the medium. Therefore, the effect of FLX in our mDPC model is probably not mediated through 5-HT reuptake inhibition, rather by its modulation of other factors, such as the ERs. Hence, our study suggests that the roles of the ER isoforms upon FLX administration should be further investigated.

For some of the genes examined (e.g., Pitx $3, N e s, E R \alpha, E R \beta$ ), we observed a non-monotonic dose-response (defined as a nonlinear relationship between dose and effect where the slope of the curve changes sign within the range of doses examined), and for other genes (e.g., $N k x 2.2, L m x 1 a, T u b b 3$ ) the effect size was greater at lower concentrations (in the nanomolar range) (Vandenberg et al., 2012). Both nonmonotonic and low-dose responses have been previously observed for chemicals that act on targets involved in endocrine regulation (including the ERs) and there is extensive literature from in vitro, in vivo, and epidemiologic studies in support of these concepts (Vandenberg et al., 2012). 


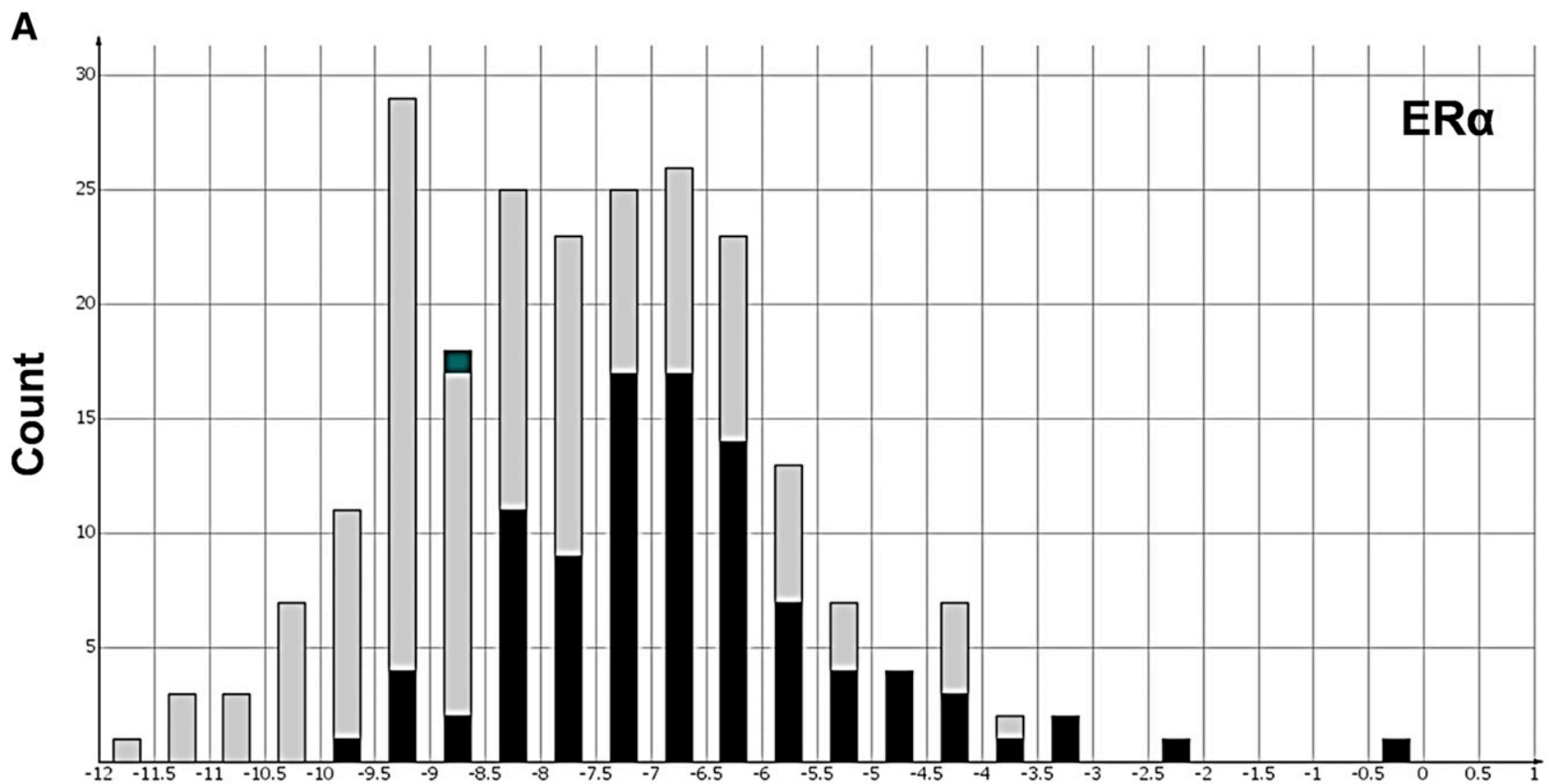

Binned docking score

B

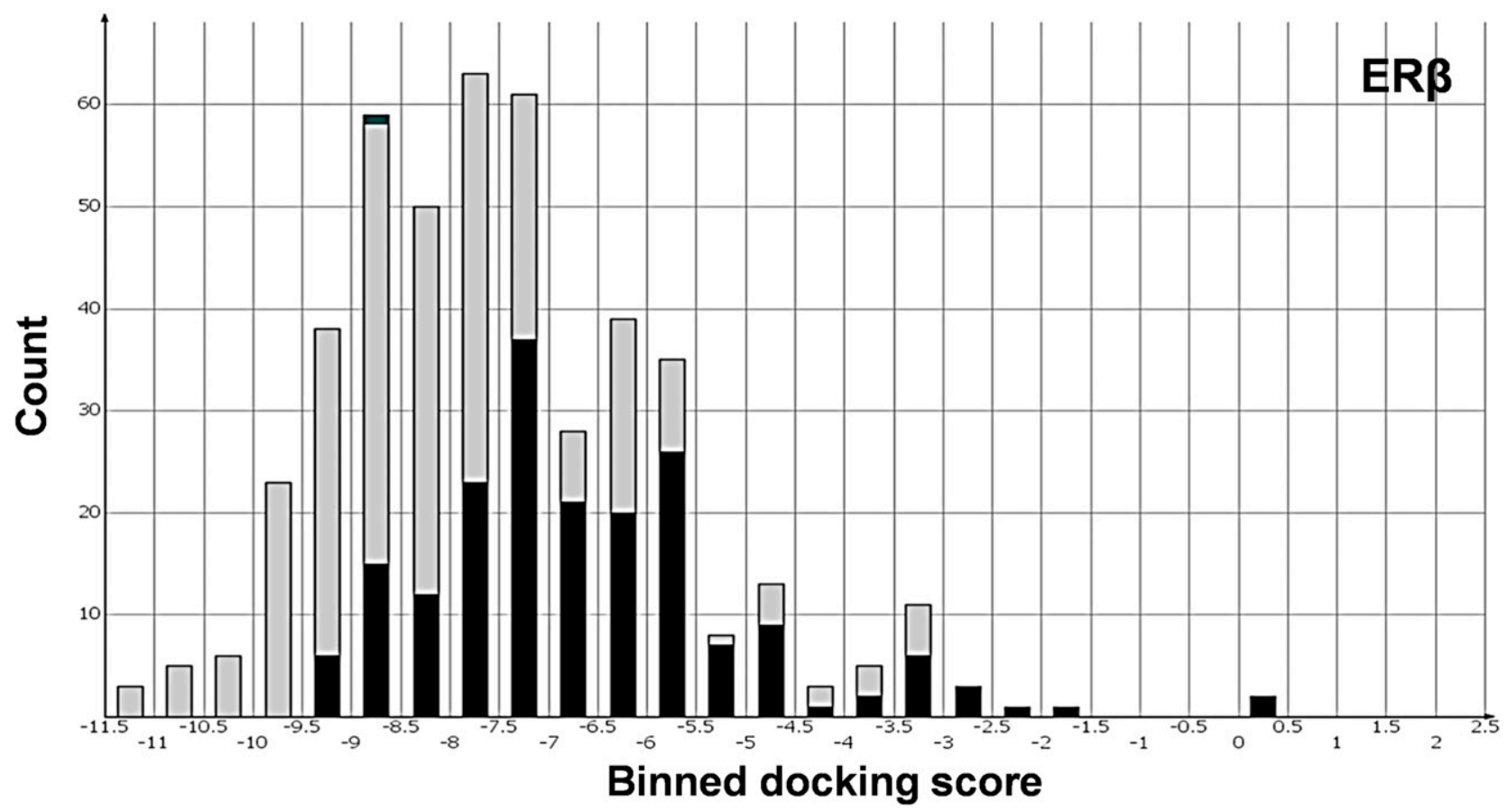

Fig. 10. Docking scores of $\mathrm{ER} \alpha(\mathrm{A})$ and $\mathrm{ER} \beta$ (B) with known active (light gray) and inactive (black) compounds in relation to FLX (dark gray).

\section{Conclusion}

In conclusion, we show here that FLX affects differentiation of dopaminergic neurons, possibly by interfering with the nuclear ERs. Our findings suggest that FLX increases neural specification toward the dopaminergic phenotype, yet decreases dopaminergic neurogenesis. This calls for further studies to establish if these molecular events are linked to the development of the dopaminergic system in humans and to address their involvement in the association between prenatal FLX exposure and increased ASD risk in children.

\section{Authorship Contributions}

Participated in research design: Lupu, Varshney, Inzunza, Nalvarte, Rüegg.

Conducted experiments: Lupu, Varshney, Mucs, Norinder, Nalvarte. Contributed new reagents or analytic tools: Inzunza, Nalvarte. 
Performed data analysis: Lupu, Varshney, Mucs, Norinders, Nalvarte.

Wrote or contributed to the writing of the manuscript: Lupu, Varshney, Mucs, Inzunza, Norinder, Loghin, Nalvarte, Rüegg.

\section{References}

Abeliovich A and Hammond R (2007) Midbrain dopamine neuron differentiation: factors and fates. Dev Biol 304:447-454.

American Psychiatric Association (2013) Autism spectrum disorder, in Diagnostic and Statistical Manual of Mental Disorders, 5th ed, pp 50-59, American Psychiatric Association, Arlington, VA.

Andalib S, Emamhadi MR, Yousefzadeh-Chabok S, Shakouri SK, Høilund-Carlsen PF, Vafaee MS, and Michel TM (2017) Maternal SSRI exposure increases the risk of autistic offspring: a meta-analysis and systematic review. Eur Psychiatry 45 $161-166$.

Arenas E, Denham M, and Villaescusa JC (2015) How to make a midbrain dopaminergic neuron. Development 142:1918-1936.

Borg I, Squire M, Menzel C, Stout K, Morgan D, Willatt L, O’Brien PC, FergusonSmith MA, Ropers HH, Tommerup N, et al. (2002) A cryptic deletion of 2q35 including part of the PAX3 gene detected by breakpoint mapping in a child with autism and a de novo 2;8 translocation. J Med Genet 39:391-399.

Bowers JM and Konopka G (2012) The role of the FOXP family of transcription factors in ASD. Dis Markers 33:251-260.

Bremer S and Hartung T (2004) The use of embryonic stem cells for regulatory developmental toxicity testing in vitro-the current status of test development. Curr Pharm Des 10:2733-2747.

Chaste P and Leboyer M (2012) Autism risk factors: genes, environment, and geneenvironment interactions. Dialogues Clin Neurosci 14:281-292.

Díaz NF, Díaz-Martínez NE, Camacho-Arroyo I, and Velasco I (2009) Estradiol promotes proliferation of dopaminergic precursors resulting in a higher proportion of dopamine neurons derived from mouse embryonic stem cells. Int J Dev Neurosci 27:493-500.

Dichter GS, Damiano CA, and Allen JA (2012) Reward circuitry dysfunction in psychiatric and neurodevelopmental disorders and genetic syndromes: animal models and clinical findings. J Neurodev Disord 4:19.

Ding D, Xu L, Fang H, Hong H, Perkins R, Harris S, Bearden ED, Shi L, and Tong W (2010) The EDKB: an established knowledge base for endocrine disrupting chemicals. BMC Bioinformatics 11 (Suppl 6):S5.

Foran CM, Weston J, Slattery M, Brooks BW, and Huggett DB (2004) Reproductive assessment of Japanese medaka (Oryzias latipes) following a four-week fluoxetine (SSRI) exposure. Arch Environ Contam Toxicol 46:511-517.

Friesner RA, Banks JL, Murphy RB, Halgren TA, Klicic JJ, Mainz DT, Repasky MP, Knoll EH, Shelley M, Perry JK, et al. (2004) Glide: a new approach for rapid, accurate docking and scoring. 1. Method and assessment of docking accuracy. $J$ Med Chem 47:1739-1749.

Gurnot C, Martin-Subero I, Mah SM, Weikum W, Goodman SJ, Brain U, Werker JF, Kobor MS, Esteller M, Oberlander TF, et al. (2015) Prenatal antidepressant exposure associated with CYP2E1 DNA methylation change in neonates. Epigenetics 10:361-372. Hallmayer J, Cleveland S, Torres A, Phillips J, Cohen B, Torigoe T, Miller J, Fedele A, Collins J, Smith K, et al. (2011) Genetic heritability and shared environmental factors among twin pairs with autism. Arch Gen Psychiatry 68:1095-1102.

Hegarty SV, Sullivan AM, and OKeeffe GW (2013) Midbrain dopaminergic neurons: a review of the molecular circuitry that regulates their development. Dev Biol 379:123-138.

Heikkinen T, Ekblad U, Palo P, and Laine K (2003) Pharmacokinetics of fluoxetine and norfluoxetine in pregnancy and lactation. Clin Pharmacol Ther 73:330-337.

James SJ, Shpyleva S, Melnyk S, Pavliv O, and Pogribny IP (2014) Elevated 5-hydroxymethylcytosine in the Engrailed-2 (EN-2) promoter is associated with increased gene expression and decreased MeCP2 binding in autism cerebellum. Transl Psychiatry 4:e460

Kaplan YC, Keskin-Arslan E, Acar S, and Sozmen K (2017) Maternal SSRI discontinuation, use, psychiatric disorder and the risk of autism in children: a metaanalysis of cohort studies. Br J Clin Pharmacol 83:2798-2806.

Khatri N, Simpson KL, Lin RCS, and Paul IA (2014) Lasting neurobehavioral abnormalities in rats after neonatal activation of serotonin $1 \mathrm{~A}$ and $1 \mathrm{~B}$ receptors: possible mechanisms for serotonin dysfunction in autistic spectrum disorders. Psychopharmacology (Berl) 231:1191-1200.

Kim J, Riggs KW, Misri S, Kent N, Oberlander TF, Grunau RE, Fitzgerald C, and Rurak DW (2006) Stereoselective disposition of fluoxetine and norfluoxetine during pregnancy and breast-feeding. Br J Clin Pharmacol 61:155-163.

Kuemerle B, Gulden F, Cherosky N, Williams E, and Herrup K (2007) The mouse Engrailed genes: a window into autism. Behav Brain Res 176:121-132.

Lammert DB and Howell BW (2016) RELN mutations in autism spectrum disorder. Front Cell Neurosci 10:84.

Lee SH, Lumelsky N, Studer L, Auerbach JM, and McKay RD (2000) Efficient generation of midbrain and hindbrain neurons from mouse embryonic stem cells. Nat Biotechnol 18:675-679.

Lister A, Regan C, Van Zwol J, and Van Der Kraak G (2009) Inhibition of egg production in zebrafish by fluoxetine and municipal effluents: a mechanistic evaluation. Aquat Toxicol 95:320-329.

Lupu D, Sjödin MOD, Varshney M, Lindberg J, Loghin F, and Rüegg J (2017) Fluoxetine modulates sex steroid levels in vitro. Clujul Med 90:420-424.

Man KK, Tong HH, Wong LY, Chan EW, Simonoff E, and Wong IC (2015) Exposure to selective serotonin reuptake inhibitors during pregnancy and risk of autism spectrum disorder in children: a systematic review and meta-analysis of observational studies. Neurosci Biobehav Rev 49:82-89.

McCarthy MM (2008) Estradiol and the developing brain. Physiol Rev 88:91-124.

Meijering E, Jacob M, Sarria JC, Steiner P, Hirling H, and Unser M (2004) Design and validation of a tool for neurite tracing and analysis in fluorescence microscopy images. Cytometry A 58:167-176.

Mennigen JA, Lado WE, Zamora JM, Duarte-Guterman P, Langlois VS, Metcalfe CD, Chang JP, Moon TW, and Trudeau VL (2010) Waterborne fluoxetine disrupts the reproductive axis in sexually mature male goldfish, Carassius auratus. Aquat Toxicol 100:354-364.

Mennigen JA, Martyniuk CJ, Crump K, Xiong H, Zhao E, Popesku J, Anisman H, Cossins AR, Xia X, and Trudeau VL (2008) Effects of fluoxetine on the reproductive axis of female goldfish (Carassius auratus). Physiol Genomics 35:273-282.

Mezzacappa A, Lasica PA, Gianfagna F, Cazas O, Hardy P, Falissard B, SutterDallay AL, and Gressier F (2017) Risk for autism spectrum disorders according to period of prenatal antidepressant exposure: a systematic review and metaanalysis. JAMA Pediatr 171:555-563.

Mysinger MM, Carchia M, Irwin JJ, and Shoichet BK (2012) Directory of useful decoys, enhanced (DUD-E): better ligands and decoys for better benchmarking. $J$ Med Chem 55:6582-6594.

Nguyen A, Rauch TA, Pfeifer GP, and Hu VW (2010) Global methylation profiling of lymphoblastoid cell lines reveals epigenetic contributions to autism spectrum disorders and a novel autism candidate gene, RORA, whose protein product is reduced in autistic brain. FASEB $J$ 24:3036-3051.

Olivier JDA, Vallès A, van Heesch F Afrasiab-Middelman A, Roelofs JJPM, Jonkers M, Peeters EJ, Korte-Bouws GAH, Dederen JP, Kiliaan AJ, et al. (2011) Fluoxetine administration to pregnant rats increases anxiety-related behavior in the offspring. Psychopharmacology (Berl) 217:419-432.

Park HR, Lee JM, Moon HE, Lee DS, Kim B-N, Kim J, Kim DG, and Paek SH (2016) A short review on the current understanding of autism spectrum disorders. Exp Neurobiol 25:1-13.

Pavăl D (2017) A dopamine hypothesis of autism spectrum disorder. Dev Neurosci 39:355-360.

Pei S, Liu L, Zhong Z, Wang H, Lin S, and Shang J (2016) Risk of prenatal depression and stress treatment: alteration on serotonin system of offspring through exposure to fluoxetine. Sci Rep 6:33822.

Perez-Caballero L, Torres-Sanchez S, Bravo L, Mico JA, and Berrocoso E (2014) Fluoxetine: a case history of its discovery and preclinical development. Expert Opin Drug Discov 9:567-578.

Pop A, Lupu DI, Cherfan J, Kiss B, and Loghin F (2015) Estrogenic/antiestrogenic activity of selected selective serotonin reuptake inhibitors. Clujul Med 88:381-385.

Rais TB and Rais A (2014) Association between antidepressants use during pregnancy and autistic spectrum disorders: a meta-analysis. Innov Clin Neurosci 11:18-22.

Rampono J, Simmer K, Ilett KF, Hackett LP, Doherty DA, Elliot R, Kok CH, Coenen A, and Forman T (2009) Placental transfer of SSRI and SNRI antidepressants and effects on the neonate. Pharmacopsychiatry 42:95-100.

Rodriguez-Porcel F, Green D, Khatri N, Harris SS, May WL, Lin RCS, and Paul IA (2011) Neonatal exposure of rats to antidepressants affects behavioral reactions to novelty and social interactions in a manner analogous to autistic spectrum disorders. Anat Rec (Hoboken) 294:1726-1735.

Sarachana T, Xu M, Wu RC, and Hu VW (2011) Sex hormones in autism: androgens and estrogens differentially and reciprocally regulate RORA, a novel candidate gene for autism. PLoS One 6:e17116.

Schultz MM, Painter MM, Bartell SE, Logue A, Furlong ET, Werner SL, and Schoenfuss HL (2011) Selective uptake and biological consequences of environmentally relevant antidepressant pharmaceutical exposures on male fathead minnows. Aquat Toxicol 104:38-47.

Schuurmans C and Guillemot F (2002) Molecular mechanisms underlying cell fate specification in the developing telencephalon. Curr Opin Neurobiol 12:26-34.

Scott-Van Zeeland AA, Dapretto M, Ghahremani DG, Poldrack RA, and Bookheimer SY (2010) Reward processing in autism. Autism Res 3:53-67.

Simpson KL, Weaver KJ, de Villers-Sidani E, Lu JY-F, Cai Z, Pang Y, Rodriguez-Porcel F, Paul IA, Merzenich M, and Lin RCS (2011) Perinatal antidepressant exposure alters cortical network function in rodents. Proc Natl Acad Sci USA 108:18465-18470.

Uzunova G, Hollander E, and Shepherd J (2014) The role of ionotropic glutamate receptors in childhood neurodevelopmental disorders: autism spectrum disorders and fragile x syndrome. Curr Neuropharmacol 12:71-98.

Vandenberg LN, Colborn T, Hayes TB, Heindel JJ, Jacobs DR Jr, Lee DH, Shioda T, Soto AM, vom Saal FS, Welshons WV, et al. (2012) Hormones and endocrinedisrupting chemicals: low-dose effects and nonmonotonic dose responses. Endocr Rev 33:378-455.

Varshney M and Nalvarte I (2017) Genes, gender, environment, and novel functions of estrogen receptor beta in the susceptibility to neurodevelopmental disorders. Brain Sci 7:E24.

Varshney MK, Inzunza J, Lupu D, Ganapathy V, Antonson P, Rüegg J, Nalvarte I, and Gustafsson J-Å (2017) Role of estrogen receptor beta in neural differentiation of mouse embryonic stem cells. Proc Natl Acad Sci USA 114:E10428-E10437.

Address correspondence to: Diana Lupu, Iuliu Haţieganu University of Medicine and Pharmacy, Pasteur street nr 6A, Cluj-Napoca, Romania; Swetox, Unit of Toxicology Sciences, Karolinska Institutet, Södertälje, Sweden. E-mail: lupu.diana@umfcluj.ro 